\title{
Impact of subject-specific musculoskeletal geometry on estimated joint kinematics, joint kinetics and muscle forces in typically developing children
}

\author{
Hans Kainz, Mariska Wesseling, Lorenzo Pitto, Antoine Falisse, Sam Van Rossom, Anja Van \\ Campenhout, Friedl De Groote, Kaat Desloovere, Chris Carty, Ilse Jonkers \\ Introduction
}

Gait analysis together with musculoskeletal modeling can be used to calculate muscle forces and assess pathological gait [1]. No generic, pediatric musculoskeletal models are available and, therefore, linear scaling methods are commonly used to personalize a generic, adult musculoskeletal model to the child's anthropometry.

\section{Research question}

How different are joint kinematics, joint kinetics and muscle force estimates of generic scaled models compared to medical-imaging based models in typically developing (TD) children?

\section{Methods}

3D motion capture data and magnetic resonance images (MRI) of a TD boy (age: 8 years; height: $1.23 \mathrm{~m}$; weight: $20.4 \mathrm{~kg}$ ) were collected. Two musculoskeletal OpenSim models were created: (1) a scaled generic model (M_gen), and (2) a MRI-based model, which included subject-specific musculoskeletal geometry (M_mri) [2]. Joint kinematics, joint kinetics and muscle forces were calculated for each model using OpenSim 3.3 [3]. Joint kinematics, joint kinetics, muscle force waveforms, as well as femoral anteversion angle, neck-shaft angle and hip joint centre location were compared between both models.

\section{Results}

Joint kinematics and joint kinetics were surprisingly similar between the $M \_g e n$ and $M \_m r i$ with rootmean-square-differences below $2.8^{\circ}$ and $0.05 \mathrm{Nm} / \mathrm{kg}$ for joint angles and moments, respectively (Figure 1 and 2). Depending on the analyzed muscle, differences in muscle forces varied substantially (up to $230 \%$ difference) between the M_gen and M_mri (Figure 3).

Femoral anteversion and neck-shaft angles differed between $M \_g e n$ and $M$ mri by 12 and 5 degrees, respectively. The hip joint centre position differed between both models by 5,15 and $6 \mathrm{~mm}$ in the anterior/posterior, superior/inferior and medial/lateral direction, respectively.

\section{Discussion}

Joint kinematics and kinetics were similar between the $M$ _gen and $M \_m r i$ but some muscle forces showed a large discrepancy between both analyzed models. This was probably caused by the different joint centre locations as well as different bony geometry, e.g. femoral anteversion and neck-shaft angle, having a major effect on the muscle moment arms.

Previous research showed that hip joint centre perturbation in the superior/inferior direction have a minor impact on joint kinematics [4], which was confirmed by our results. However, depending on the muscle, a difference of joint centre location of $15 \mathrm{~mm}$ in the superior/inferior direction can have a large impact on the moment arm and, therefore, significantly changing the magnitude of the muscle force. 
Considering femoral anteversion angle changes around 15 degrees and the neck-shaft angle around 6 degrees during growth [5] and our observed differences between the $M_{-}$gen and $M \_m r i$, pediatric musculoskeletal models, which include age-specific femoral geometry, are needed to improve muscle force estimations in the absence of medical-imaging based models.

\section{References}

[1] Hoang \& Reinbolt, Gait\&Posture.36,2012,405-8.

[2] Scheys, et al. Gait\&Posture.33,2011,158-164.

[3] Delp, et al. IEEE Trans.Biomed.Eng.54,2007,1940-1950.

[4] Kainz, et al. Gait\&Posture.57,2017,154-160.

[5] Bobroff, et al. Clin.Orthop.Relat.Res.,1999,194-204. 

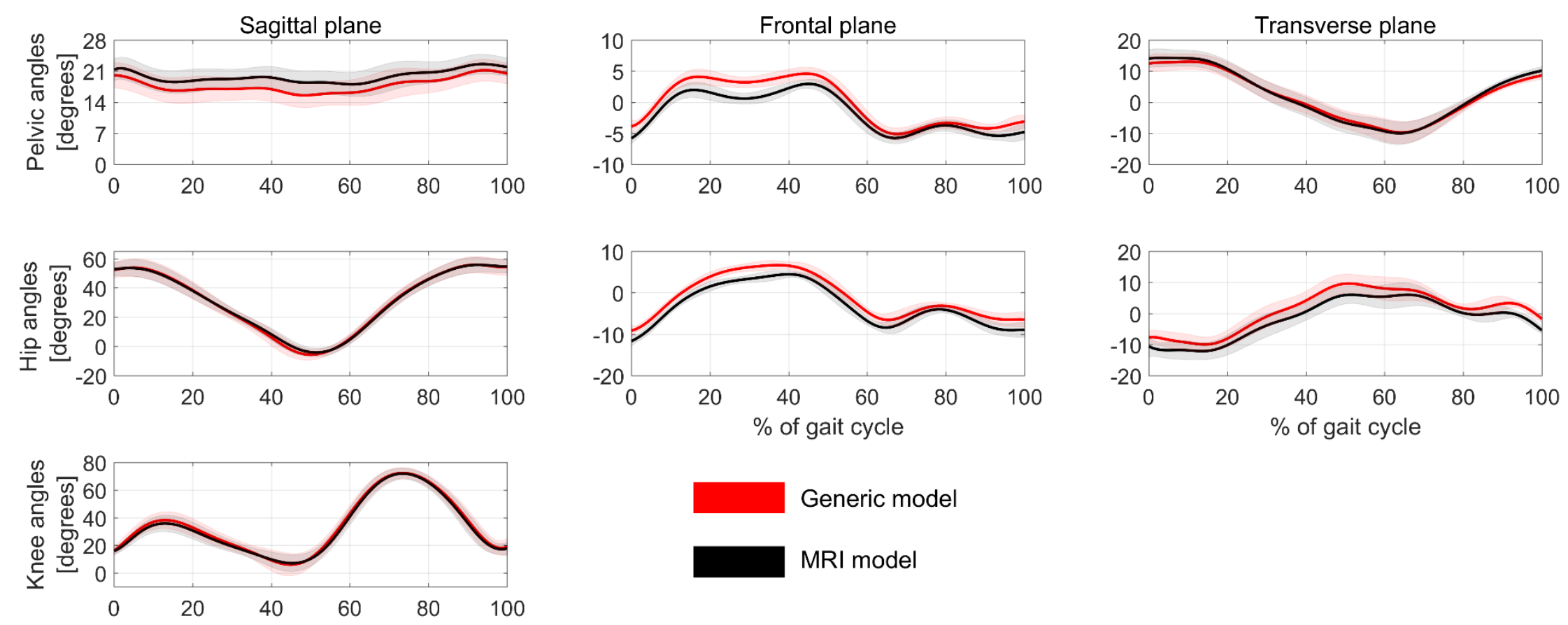

Generic model

\section{MRI model}

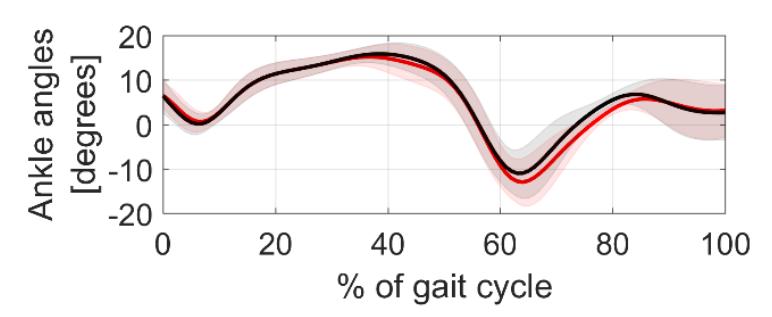

Figure 1. Joint kinematics 

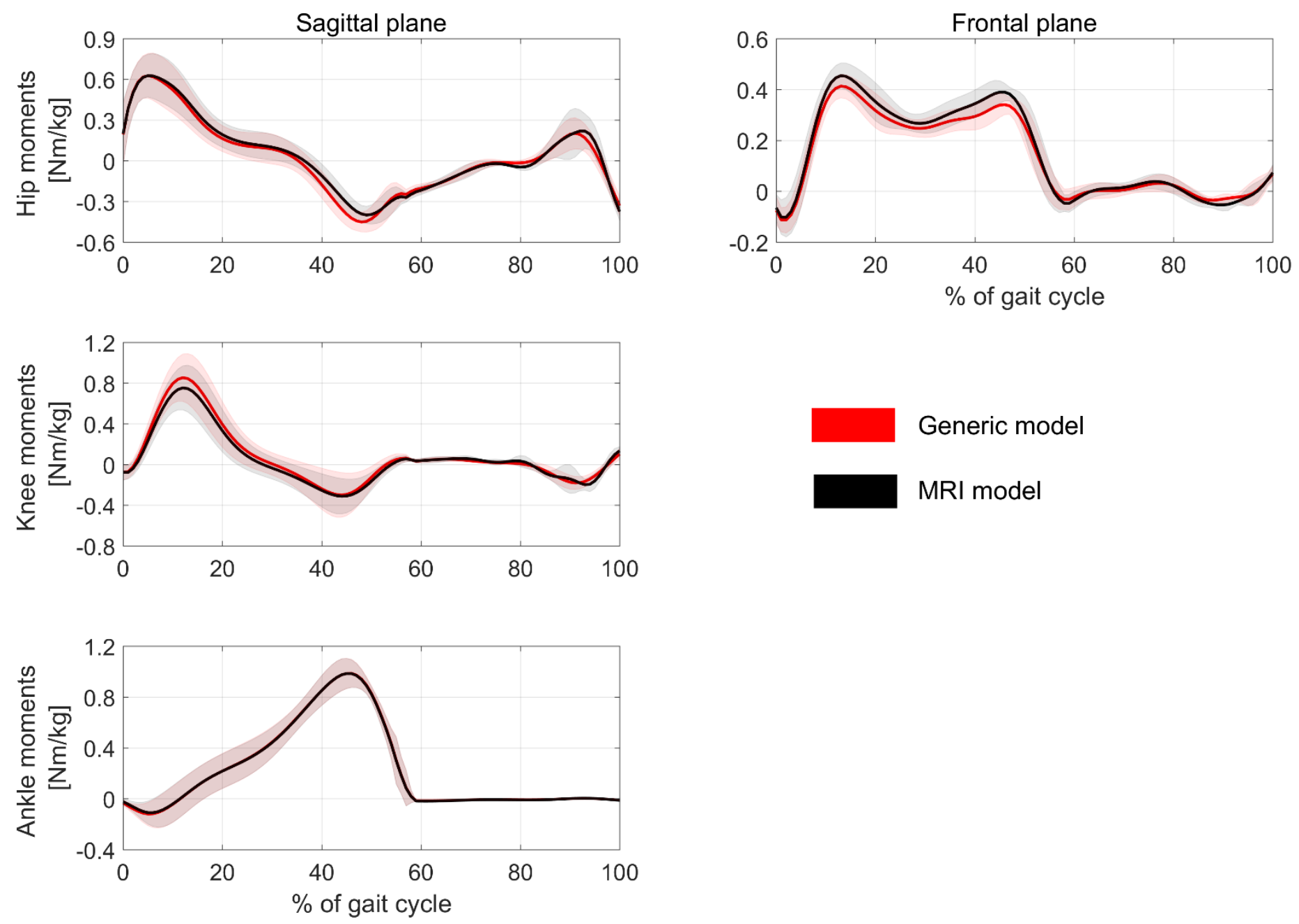

Figure 2. Joint kinetics 

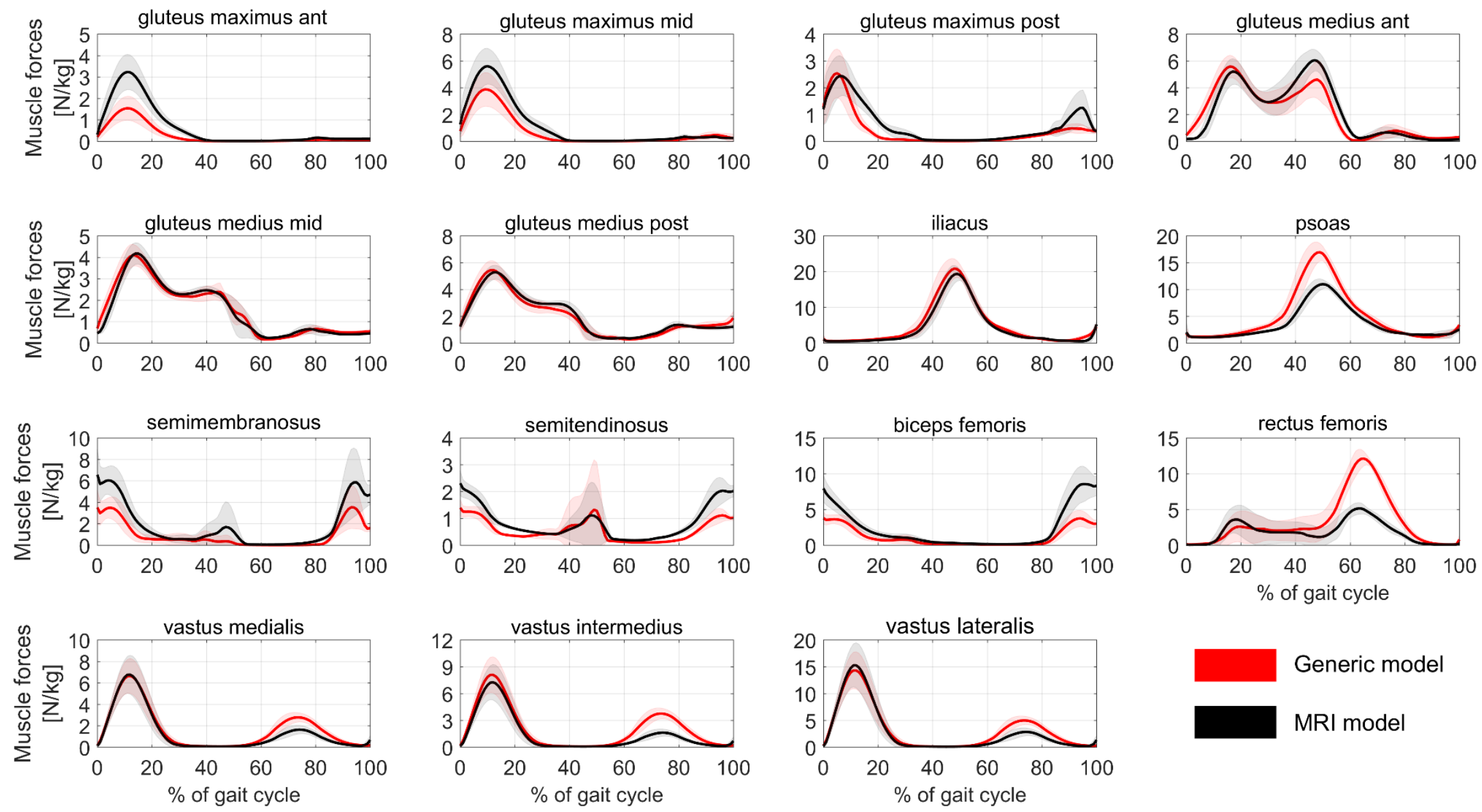

Figure 3. Muscle forces during gait 